\title{
Functional Brain Network Learning Based on Spatial Similarity for Brain Disorders Identification
}

\author{
Lei Sun, Tingting Guo \\ School of Mathematics Science, Liaocheng University, Liaocheng, China \\ Email: sunleizy1988@163.com, guotingting1014@163.com
}

How to cite this paper: Sun, L. and Guo, T.T. (2020) Functional Brain Network Learning Based on Spatial Similarity for Brain Disorders Identification. Journal of Applied Mathematics and Physics, 8, 2427-2437.

https://doi.org/10.4236/jamp.2020.811179

Received: October 16, 2020

Accepted: November 15, 2020

Published: November 18, 2020

Copyright $\odot 2020$ by author(s) and Scientific Research Publishing Inc. This work is licensed under the Creative Commons Attribution International License (CC BY 4.0).

http://creativecommons.org/licenses/by/4.0/

(c) (i) Open Access

\begin{abstract}
Functional brain network (FBN) measures based on functional magnetic resonance imaging (fMRI) data, has become important biomarkers for early diagnosis and prediction of clinical outcomes in neurological diseases, such as Alzheimer's diseases (AD) and its prodromal state (i.e., Mild cognitive impairment, MCI). In the past decades, researchers have developed numbers of approaches for FBN estimation, including Pearson's correction (PC), sparse representation (SR), and so on. Despite their popularity and wide applications in current studies, most of the approaches for FBN estimation only consider the dependency between the measured blood oxygen level dependent (BOLD) time series, but ignore the spatial relationships between pairs of brain regions. In practice, the strength of functional connection between brain regions will decrease as their distance increases. Inspired by this, we proposed a new approach for FBN estimation based on the assumption that the closer brain regions tend to share stronger relationships or similarities. To verify the effectiveness of the proposed method, we conduct experiments on a public dataset to identify the patients with MCIs from health controls (HCs) using the estimated FBNs. Experimental results demonstrate that the proposed approach yields statistically significant improvement in seven performance metrics over using the baseline methods.
\end{abstract}

\section{Keywords}

Functional Brain Network, Pearson's Correction, Sparse Representation, Spatial Relationships, Similarity, Mild Cognitive Impairment

\section{Introduction}

As the common neurological diseases, Alzheimer's disease (AD) is defined as 
diagnostic criteria of progressive cognitive and memory impairment [1]. It is reported that increasing populations will suffer from $\mathrm{AD}$ over time, which will severely affect their health and daily life [2]. Consequently, it is urgently needed for early detection and correct diagnosis for mild cognitive impairment (i.e., $\mathrm{MCI}$, the early prodromal stage of $\mathrm{AD}$ ) populations [3].

As a widely used non-invasive technique for measuring brain activities, functional magnetic resonance imaging (fMRI) has been successfully applied to explore early MCI diagnosis [4]. In particular, the popular diagnosis models include Bayesian network (BN) [5], support vector machine (SVM) [6], and graph learning (GL) [7], but, due to the randomness and the asynchronization of the spontaneous brain activities, it is difficult to train these models directly using the fMRI data. In contrast, functional brain network (FBN), estimated by fMRI data, can provide an effective tool to better understand and study the brain structure of neurological diseases (i.e., MCI) [8]. In other words, estimating a high-quality FBN may be helpful for the subsequent neurological identification task [9] [10].

Mathematically, each node in the FBN corresponds to the brain region of interest (ROI), and each edge shows the dependency relationships between pairs of ROIs. To estimate these edges, researchers have proposed numbers of approaches, in which the standard correction-based approaches include Pearson's correction (PC)-based approach [11], (regularized) partial correction-based approach [12], and dynamic causal model [13] respectively. In this paper, we only consider second-order approaches, i.e., PC-based method and (regularized) partial correction-based method, since a recent study has empirically validated that they are more effective than many complex higher-order approaches.

Despite its simplicity and popularity, PC always produces dense FBNs that all nodes are fully-connected by edges, since it is sensitive to both direct and indirect functional connections in the brain. Thus, PC-based FBNs generally contain noisy or uninformative information. To remove the spurious information from the estimated FBNs, a common strategy is thresholding [14]. However, the optimal value of thresholding is currently an open problem. In contrast, partial correction is only sensitive to direct connections that can explain more complex interactions among brain regions. Even so, the estimation of partial correction matrix is usually ill-posed due to the singularity of the sample covariance matrix. To over this problem, the regularization term is generally contained the partial correction model, which generates a sparse representation (SR)-based method. Similarly, it is difficult to select the optimal value for the regularization term. In addition, both the above methods do not consider the spatial relationships of the BOLD time series.

In practice, according to previous work, the strength of functional connection between two ROIs will decrease as their distance increases. For instance, people in a society are analogous as a node (or ROI), and the social relationship between people is analogous as edge (or functional connectivity) in the estimated FBN [15]. If two persons are spaced close together, they may have similar rela- 
tionships or social circles. Thus, we simply assume that the brain regions close to each other tend to share stronger relationships or similarity. Inspired by this assumption, we propose a new approach for FBN estimation based on the spatial distance between ROIs. To verify the effectiveness of our model, we utilize the FBNs estimated by our method to identify the patients with MCI from health controls (HCs). Experiments conducting on a public dataset demonstrate that the proposed model outperforms than baseline methods (i.e., PC and SR). To better understand, we describe the whole pipeline of MCI classification task used in this study in Figure 1 that contains three main steps: 1) Data Acquisition, 2) FBN Estimation, and 3) Classification, which will be shown in Section II, III, and IV respectively.

The rest of this paper is organized as follows: in Section 2, we introduce the data sources and preprocessing step; in Section 3, we review several related works and develop our method for FBN estimation; in Section 4, we describe the experimental setting and verify the proposed method for MCI identification task; in Section V, we conclude this paper.

\section{Data Acquisition}

In this section, ADNI (http://adni.loni.ucla.edu) dataset was utilized to identify the participants with MCI from HCs based on the estimated FBNs to demonstrate the effectiveness of the proposed method. Concretely, for MCI vs. NC classification task, the preprocessed dataset with 137 subjects (68 MCIs and 69 HCs) was utilized in this study, in which all participants were scanned by $3.0 \mathrm{~T}$ Philip scanners. Further, to remain the signal stabilization, the first three volumes of each participant were removed from the fMRI time series/BOLD signals. Then, the remaining volumes were processed by Data Processing Assistant for Resting-State fMRI (DPARSF) toolbox [16]. The preprocessing step can be summarized into the following steps: 1) head motion correction (i.e., subjects with head motion larger than $2 \mathrm{~mm}$ or $2^{\circ}$ were excluded), 2) Friston 24-parameters nuisance regression model (i.e., the influence of the ventricle, white matter signals, and the high-order effect of head motion were removed ); 3) registration to standard Montreal Neurological Institute (MNI) space; 4) spatially smoothing and temporal band-pass filtering $(0.01-0.1 \mathrm{~Hz})$. Finally, according to the automated anatomical labeling (AAL) atlas [17], the brain was divided into 116 ROIs, and all BOLD time series of the whole ROI was placed into $X \in R^{137 \times 116}$.
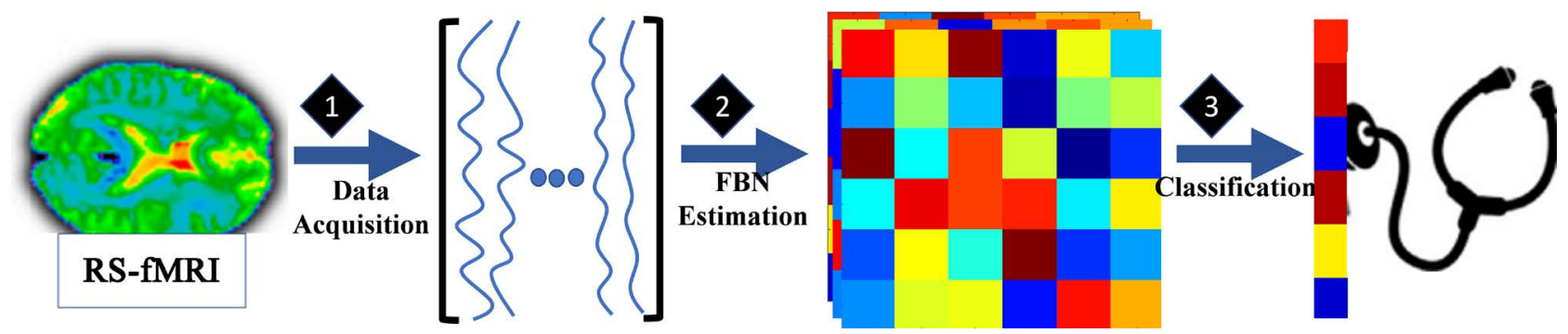

Figure 1. The classification pipeline used in our study. 


\section{FBN Estimation}

\subsection{Pearson's Correlation}

PC, as the most popular and simplest method to estimate FBNs, can be defined as follows mathematically:

$$
S_{i j}=\frac{\left(x_{i}-\bar{x}_{i}\right)^{T}\left(x_{j}-\bar{x}_{j}\right)}{\sqrt{\left(x_{i}-\bar{x}_{i}\right)^{T}\left(x_{i}-\bar{x}_{i}\right)} \sqrt{\left(x_{j}-\bar{x}_{j}\right)^{T}\left(x_{j}-\bar{x}_{j}\right)}},
$$

where $x_{i} \in R^{T}, \forall i=1, \cdots, m$ is the mean time series extracted from th brain region, $T$ is the number of time points in each series, $m$ is the total number of ROIs, $\bar{x}_{i} \in R^{T}$ is the mean of $x_{i}$, and $S_{i j}, \forall i, j=1, \cdots, m$ is the connection weight between $i$ th ROI and $j$ th ROI. In particular, we redefine $x_{i}=\frac{\left(x_{i}-\bar{x}_{i}\right)}{\sqrt{\left(x_{i}-\bar{x}_{i}\right)^{T}\left(x_{i}-\bar{x}_{i}\right)}}$, i.e., the new $x_{i}$ has been centralized by $x_{i}-\bar{x}_{i}$ and normalized by $\sqrt{\left(x_{i}-\bar{x}_{i}\right)^{T}\left(x_{i}-\bar{x}_{i}\right)}$. Finally, Equation (1) can be simplified into $S_{i j}=x_{i}^{T} x_{j}$, which corresponds to the optimal solution of the following form:

$$
\min _{S_{i j}} \sum_{i, j=1}^{m}\left\|x_{i}-S_{i j} x_{j}\right\| .
$$

Equivalently, Equation (2) can be further transformed into the following matrix model:

$$
\min _{S}\left\|S-X^{T} X\right\|_{F}^{2},
$$

where $S$ is the connectivity matrix between ROIs, $X=\left[x_{1}, x_{2}, \cdots, x_{i}, \cdots, x_{m}\right] \in R^{T \times m}$ is the data matrix, and $\|\cdot\|_{F}$ represents the $F$-norm of a matrix. In fact, FBN estimated by PC is very dense that contains noises or redundant connections. Thus, the threshold strategy is utilized to remove these redundant connections. However, selecting the optimal value of the threshold is still difficult.

\subsection{Sparse Representation}

As an alternative PC, SR is one of the commonly-used approaches to calculate the partial correction, which can estimate more reliable relationships between two ROIs by regressing out the confounding effect from other ROIs. The mathematical model of SR can be defined below:

$$
\min _{S_{i j}} \sum_{i=1}^{m}\left\|x_{i}-\sum_{j \neq i} S_{i j} x_{j}\right\|^{2}+\lambda \sum_{j \neq i}\left|S_{i j}\right|,
$$

Accordingly, it can be further rewritten by the following matrix form:

$$
\begin{aligned}
& \min _{S}\|X-X S\|_{F}^{2}+\lambda\|S\|_{1} \\
& \text { s.t. } S_{i i}=0, \forall i=1, \cdots, m
\end{aligned}
$$

where $\|\cdot\|_{1}$ shows $l_{1}$-norm of a matrix. The constraint $S_{i i}=0$ is utilized here to avoid the trivial solution (i.e., $S=I$, the identity matrix) by removing $x_{i}$ from $X$. It is worth noting that the optimized matrix $S$ in Equation (5) is asymmetric. To be consistent with PC, SR-based FBNs (i.e., S) can be defined as 
$S=\left(S+S^{T}\right) / 2$. It can be observed that the data fitting term of Equation (5) can obtain the sparse FBNs, since the data fitting term estimates the partial corrections between two ROIs, and the regularization parameter plays an important role to control the sparsity of the estimated FBNs. However, this method ignores the spatial relationship of pairs of time series.

\subsection{The Proposed Method}

\section{Model}

The proposed method can be described by the following optimization model:

$$
\begin{aligned}
& \min _{S_{i j}} \sum_{i, j=1}^{m}\left\|x_{i}-x_{j}\right\|^{2} S_{i j} \\
& \text { s.t., } S_{i j} \geq 0, \sum_{j=1}^{m} S_{i j} \geq 1, \forall i=1, \cdots, m
\end{aligned}
$$

Equally, Equation (6) can be rewritten by the following matrix form in mathematic:

$$
\begin{aligned}
& \min _{S} \operatorname{tr} X H X^{T} \\
& \text { s.t., } S_{i j} \geq 0, \sum_{j=1}^{m} S_{i j} \geq 1, \forall i=1, \cdots, m
\end{aligned}
$$

where $H=D-S$ shows the Laplacian matrix, $D$ is a diagonal matrix with diagonal elements $\sum_{j=1}^{m} S_{i j} \geq 1, \forall i=1, \cdots, m$, the constraint $S_{i j} \geq 0, \sum_{j=1}^{m} S_{i j} \geq 1$ in Equation (7) is to prevent trivial solutions (i.e., $S_{i j}=0, \forall i, j=1, \cdots, m$ ). To our best knowledge, the brain is not a fully connected network. To estimate the sparse FBNs, we can utilize the following step: 1) deciding the number of edges of estimated FBNs based on utilizing $k$-nearest neighbor ( $k$-NN) [18], that is, the brain region $i$ and $j$ are connected by an edge if $i$ is among $k$ nearest neighbors of $j$ or $j$ is among $k$ nearest neighbors of $i$;) choosing the weights to edges between ROIs based on Equation (7) reasonably. In particular, the brain region $i$ and $j$ are spatially connected close together, they will share the stronger similarity. In contrast, they will obtain the small weight. In Table 1, we summarize the main algorithm for solving the proposed FBN estimation methods.

\section{Experimental Results}

\subsection{Experimental Setting}

In our paper, we utilize PC and SR as the baseline methods to compare our method. Note that, the proposed method constrains the edge weights to be nonnegative. Thus, besides PC and SR, we also select PC+ and SR+ as the baseline methods based on the original PC and SR. In particular, PC+ and SR+ only keep the positive edges and turn the negative edges into zero in PC and SR. Since the threshold, regularization parameter or the number of nearest neighbor involved in both five methods (i.e., $\mathrm{PC}, \mathrm{PC}+, \mathrm{SR}, \mathrm{SR}+$ and ours) play a significant impact on the final classification accuracy, we set the threshold corresponding to different sparsity level in the set of $[0,10 \% \ldots, 90 \%, 99 \%]$ for PC-based method, in which each percentage represents the proportion of edges that are removed, set the regularization parameter in the range of $\left[2^{-5}, 2^{-4}, \cdots, 2^{4}, 2^{5}\right]$ for SR-based 
Table 1. The optimization algorithm of the proposed model.

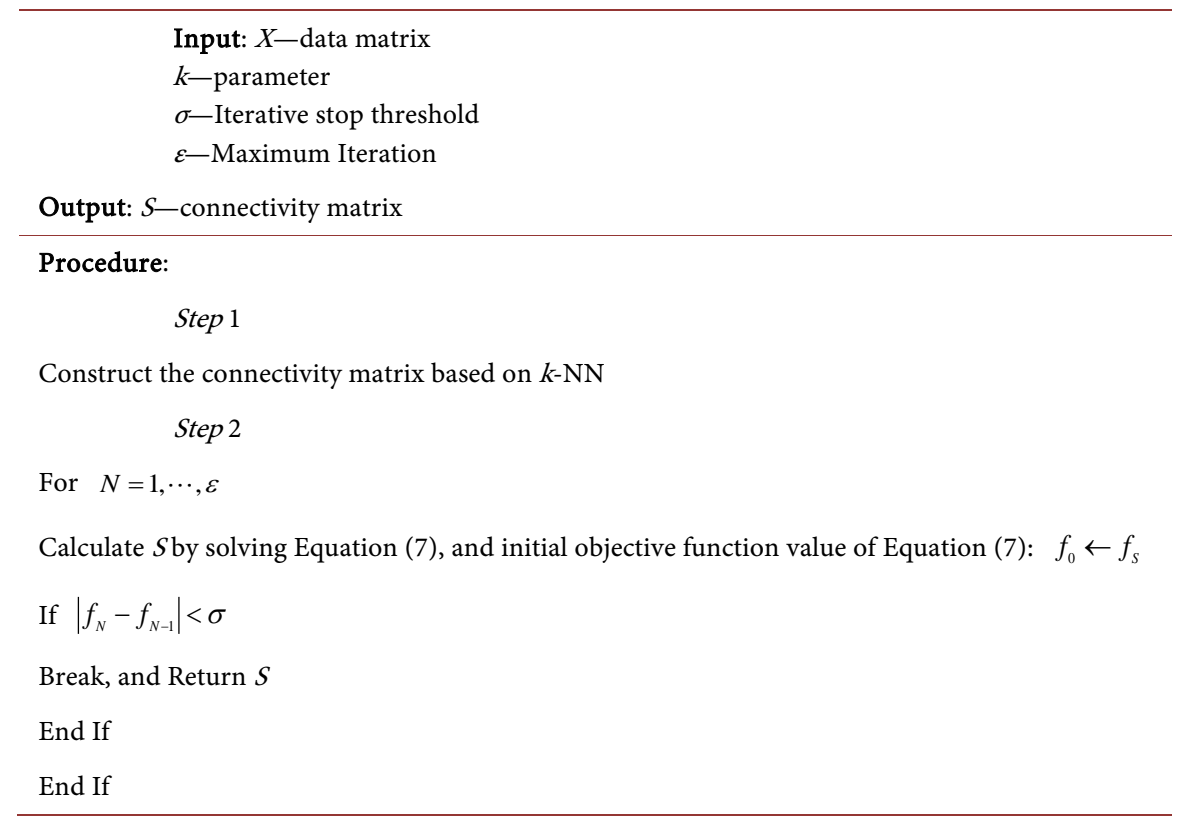

methods, and search the nearest neighbor in the range of $[10,12, \cdots, 28,30]$.

However, numbers of features (or functional connectivity) from the constructed FBNs is larger than the amounts of participants. In fact, not all features contribute to the diagnosis labels and even may capture redundant information already uncovered by other features. Therefore, to remove irrelevant features not contributing to the prediction power of the model and achieving higher identification accuracy rates, we apply the simplest $t$-test with four accepted $p$-values $(0.001,0.005,0.01,0.05)$ for feature selection and choose the linear support vector machine (SVM) with default parameter $\mathrm{C}=1$ to conduct the subsequent classification task. Further, we choose leave-one-out cross validation (LOOCV) to evaluate the performance of involved methods. Finally, we utilize a series of statistical measurement indices, including accuracy (ACC), sensitivity (SEN), specificity (SPE), balanced accuracy (BAC), positive predictive value (PPV), negative predictive value (NPV) and the area under the receiver operating characteristic (ROC) curve (AUC) to evaluate the classification performance of different methods. In particular, their definitions are shown as follows:

$\mathrm{ACC}=(\mathrm{TP}+\mathrm{TN}) /(\mathrm{TP}+\mathrm{FP}+\mathrm{TN}+\mathrm{FN}), \quad \mathrm{SEN}=\mathrm{TP} /(\mathrm{TP}+\mathrm{FN})$, $\mathrm{SPE}=\mathrm{TN} /(\mathrm{TN}+\mathrm{FP}), \quad \mathrm{BAC}=(\mathrm{SEN}+\mathrm{SPE}) / 2, \quad \mathrm{PPV}=\mathrm{TP} /(\mathrm{TP}+\mathrm{FP})$, $\mathrm{PPV}=\mathrm{TP} /(\mathrm{TP}+\mathrm{FP})$ and $\mathrm{NPV}=\mathrm{TN} /(\mathrm{TN}+\mathrm{FN})$, where $\mathrm{TP}, \mathrm{TN}, \mathrm{FP}$ and $\mathrm{FN}$ indicate the true positive, true negative, false positive and false negative.

\subsection{FBN Estimation}

In this section, we take one of the participants from ADNI dataset as an example to visualize the FBNs estimated by five different methods (i.e., PC, PC+, SR, SR+ and ours). In Figure 2, we show the experimental results, in which the thresholds or regularization parameters involved in PC- and SR-based methods are 


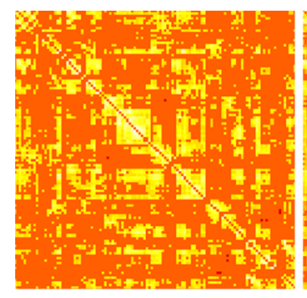

PC

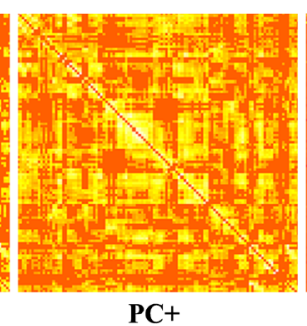

PC+

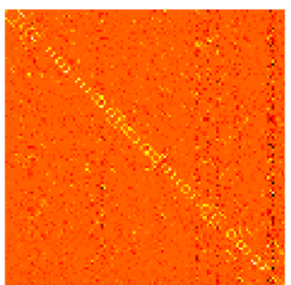

SR

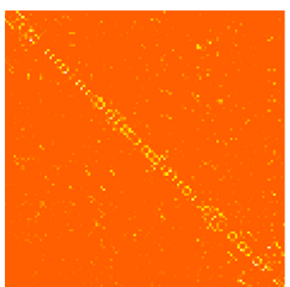

SR+

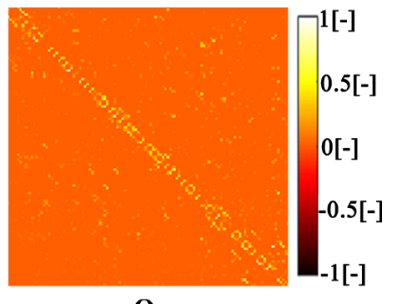

Ours

Figure 2. The FBN construction estimated by five methods. Note that, the adjacency matrices of FCNs constructed by five different methods (i.e., PC, PC+, SR, SR+ and ours), in which the elements have been normalized into interval of $[-1,1]$ for the convenience of comparison. In particular, PC+ and SR+ only keep the positive edges in PC and SR, and turn their negative edges into zero, respectively.

determined based on their best classification accuracy. Specifically, the thresholds used in PC and PC+ are 50\% and 40\%, while the values of the regularization parameter or nearest neighbor used in SR and SR+ are $2^{2}, 2^{5}$ and 22 , respectively. It can be visualized in Figure 2 that PC-based FBNs produces dense FBN easily. The reason for this is that the full correction is utilized to model the network adjacency matrix, and thus generates false functional connections. Although FBN estimated by PC+ improves the sparsity, it is still denser than SR and SR+-based FBN. That is because the original PC contains more redundant connections. In contrast, due to the introduction of regularization term, the estimated FBN by SR and SR+ are sparse. Compared with the baseline methods, the FBN estimated by the proposed method looks very clean, and its topological structure is similar to that of SR and SR+-based FBNs. We can argue that the spatial relationship between ROIs plays an important role in FBN estimation.

\subsection{Classification Results}

In Figure 3, we report the quantitative results achieved by five different methods under four accepted $p$-values in MCI classification tasks. According to the experimental results, we could have three main observations. First, the proposed method generally achieves better classification performance, compared with the baseline methods (i.e., PC, PC+, SR and SR+). For instance, in light of the average ACC values under four accepted $p$-values (i.e., 0.001, 0.005, 0.01, 0.05), the proposed method achieves the values of $80.29 \%, 81.75 \%, 87.59 \%$ and $88.32 \%$, which largely outperforms the best results of baseline methods. These results suggest that the proposed method could perform better classification performance. Second, in terms of SEN values under four $p$-values, the proposed method also obtains the highest classification results, indicating that the proposed method may have a practically meaningful advantage for timely diagnosis of MCI population. For the remaining metrics, the final results also show the effectiveness of the proposed method.

\subsection{Sensitivity to Network Modelling Parameters}

In practice, the final classification accuracies are affected not only by $p$-values, but also by different network modelling parameters (e.g., thresholds and regula- 
rization parameters). In order to investigate the influence of parameters on the final classification accuracy, we conduct MCI classification experiments, and report their results with different values of thresholds or regularization parameters under four accepted $p$-values in Figure 4. It can be observed that the results based on the baseline methods will significantly influence the parametric values.

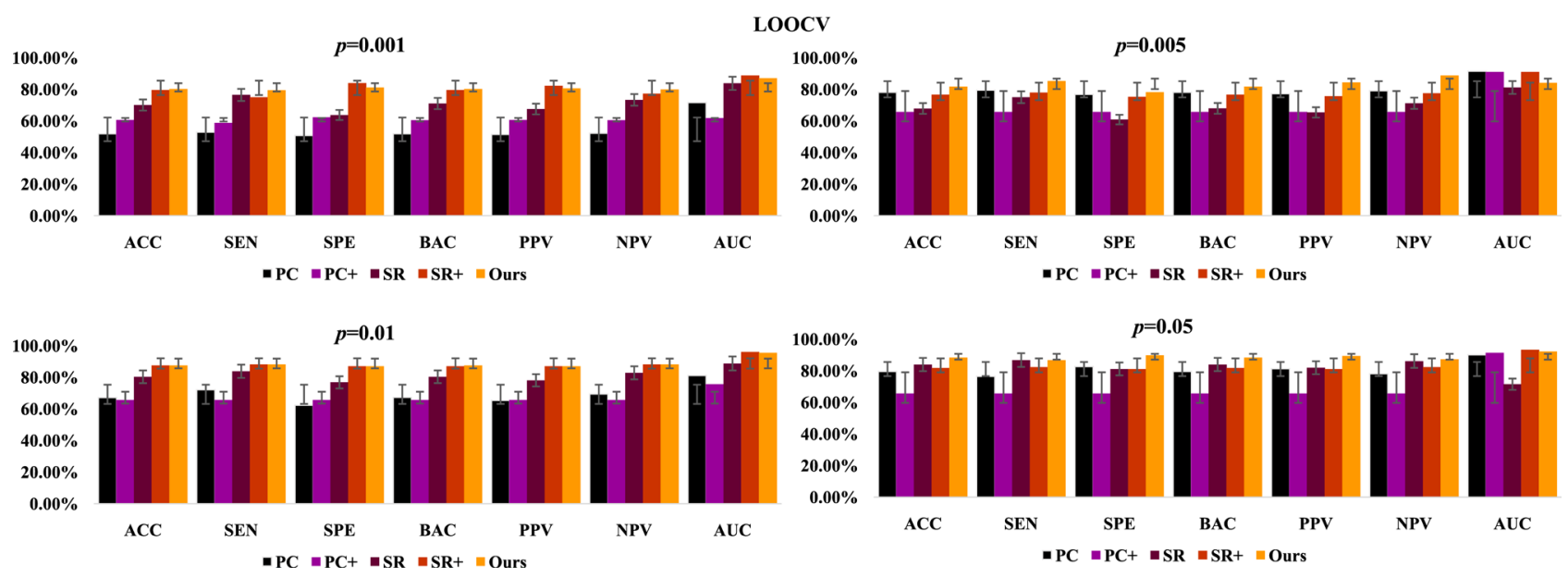

Figure 3. The classification results based on seven performance indices by five different methods. In particular, each subplot represents the classification results based on five methods with a different threshold under seven performance indices, where the vertical axis represents the average classification results.
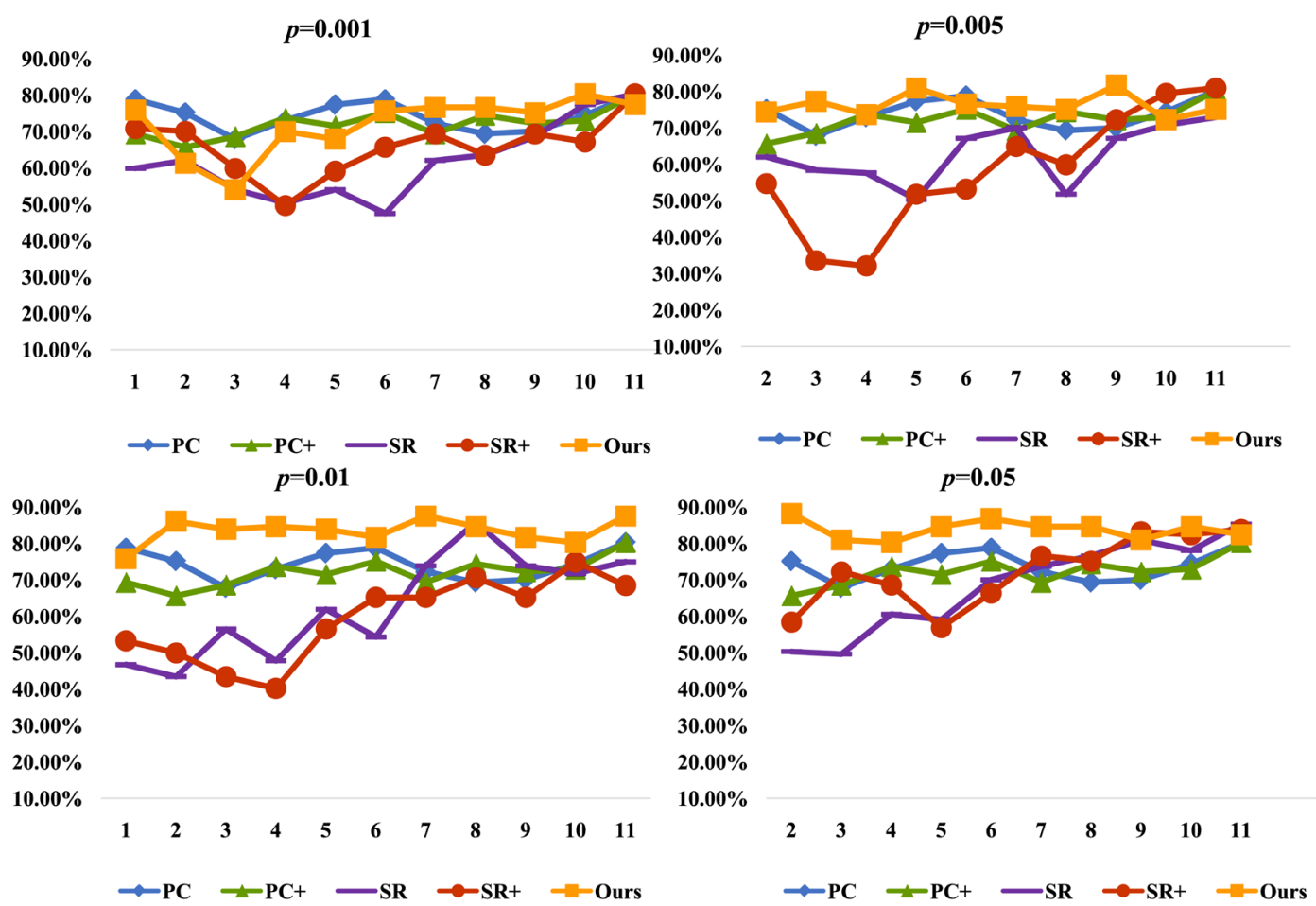

Figure 4. The MCI classification accuracies based on five FBN estimation methods (i.e., PC, PC+, SR, SR+ and ours) with different network modelling parametric values are shown in this figure. Note that, the horizontal axis for PC-based, SR-based, and our methods represents different thresholds, the regularization parameters, and for SR-based methods show while the vertical axis for five methods represents the average classification. 


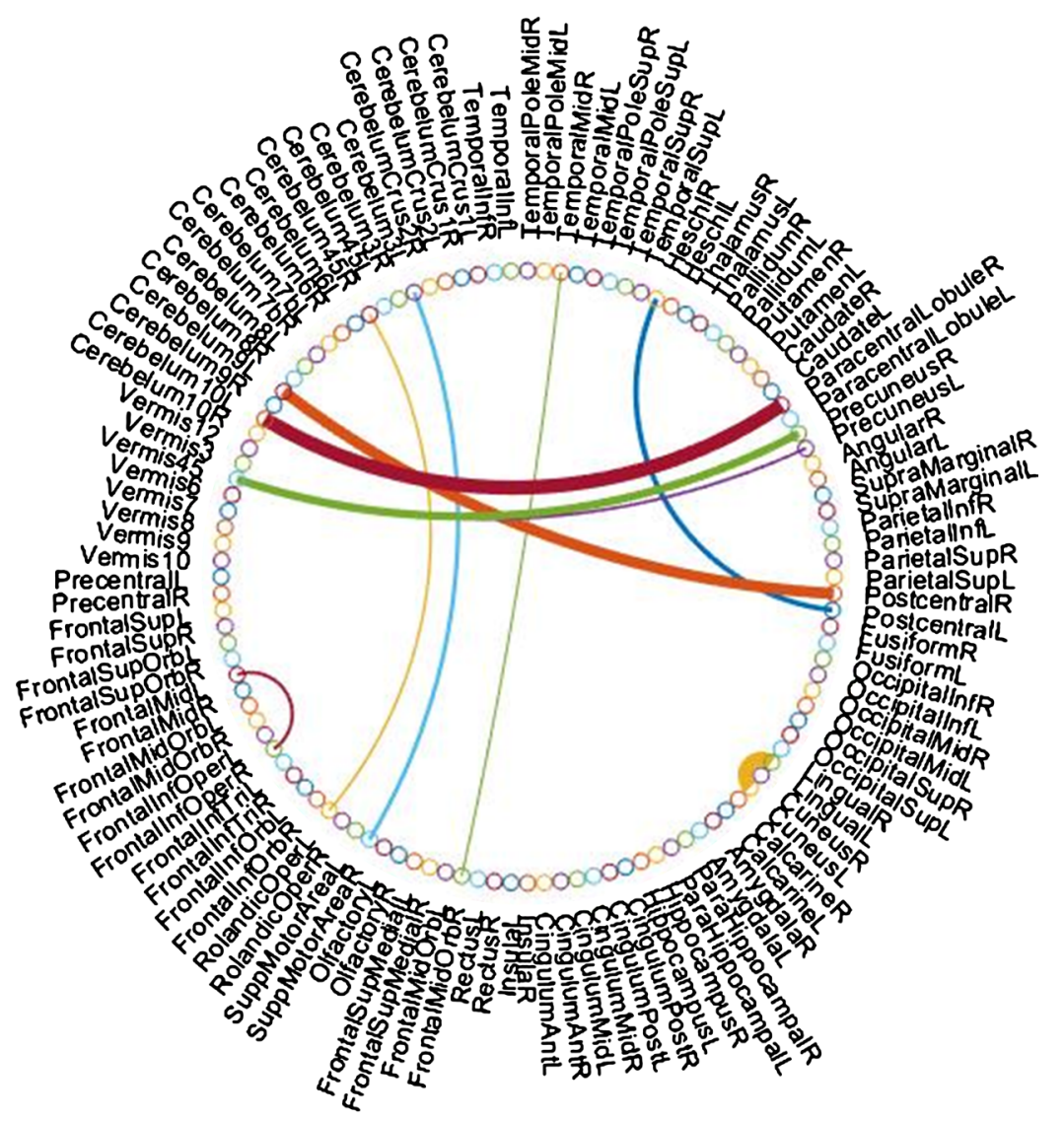

Figure 5. The discriminative features selected by $t$-test $(p<0.001)$ between MCI vs. NC classification task based on AAL atlas for brain parcellation. In particular, this figure is created by a specific function (i.e., Circular Graph) based on Matlab software).

In contrast, the proposed method can obtain the higher classification results, compared with the competing methods in most cases, indicating the results of our method is reliable.

\subsection{Discriminative Features}

To visualize which features (i.e., functional connections) contribute more to the final classification task, we select the top 10 most discriminative features for identifying MCI population based on $t$-test for feature selection, and show the results in Figure 5. In particular, the thickness of each arc shown in Figure 5 represents the discriminative power that is inversely proportional to corresponding to $p$-value. In addition, according to these features, we further find several of them correspond to the brain regions, such as the left postcentral region [19], the right precuneus region [20], are reported as potential biomarkers for MCI vs. NC classification task. The results further prove the effectiveness of the proposed method.

\section{Conclusion}

In this paper, PC and SR are utilized as the baseline methods to estimate FBNs. 
PC is sensitive to the direct and indirect relationship between pairs of ROIs, and thus always produces false functional connections. In contrast, SR can construct sparse FBN based on the obtained BOLD time series, due to the introduction of the regularization term. Even so, this method also ignores the spatial relationship between pairs of time series. Inspired by this, we propose a novel approach based on the spatial distance, and conduct MCI vs. NC classification task to verify the effectiveness of our method. Experimental results show that the proposed method can obtain better accuracy than the baseline methods. In the future, we will propose more novel FBN estimation methods corresponding to practical questions.

\section{Conflicts of Interest}

The authors declare no conflicts of interest regarding the publication of this paper.

\section{References}

[1] Wee, C.-Y., et al. (2012) Identification of MCI Individuals Using Structural and Functional Connectivity Networks. NeuroImage, 59, 2045-2056.

https://doi.org/10.1016/j.neuroimage.2011.10.015

[2] Grundman, M., et al. (2004) Mild Cognitive Impairment Can Be Distinguished from Alzheimer Disease and Normal Aging for Clinical Trials. Archives of Neurology, 61, 59-66. https://doi.org/10.1001/archneur.61.1.59

[3] Misra, C., Fan, Y. and Davatzikos, C. (2009) Baseline and Longitudinal Patterns of Brain Atrophy in MCI Patients, and Their Use in Prediction of Short-Term Conversion to AD: Results from ADNI. NeuroImage, 44, 1415-1422. https://doi.org/10.1016/j.neuroimage.2008.10.031

[4] Huettel, S.A., Song, A.W. and McCarthy, G. (2004) Functional Magnetic Resonance Imaging. Sinauer Associates, Sunderland.

[5] Friedman, N., Geiger, D. and Goldszmidt, M. (1997) Bayesian Network Classifiers. Machine Learning, 29, 131-163. https://doi.org/10.1023/A:1007465528199

[6] Suykens, J.A. and Vandewalle, J. (1999) Least Squares Support Vector Machine Classifiers. Neural Processing Letters, 9, 293-300. https://doi.org/10.1023/A:1018628609742

[7] Wu, Z., Pan, S., Chen, F., Long, G., Zhang, C. and Philip, S.Y. (2020) A Comprehensive Survey on Graph Neural Networks. IEEE Transactions on Neural Networks and Learning Systems. https://doi.org/10.1109/TNNLS.2020.2978386

[8] Dickerson, B.C. and Sperling, R.A. (2009) Large-Scale Functional Brain Network Abnormalities in Alzheimer's Disease: Insights from Functional NeuroImaging. Behavioural Neurology, 21, 63-75. https://doi.org/10.1155/2009/610392

[9] Baggio, H.C., et al. (2014) Functional Brain Networks and Cognitive Deficits in Parkinson's Disease. Human Brain Mapping, 35, 4620-4634.

https://doi.org/10.1002/hbm.22499

[10] Seo, E.H., et al. (2013) Whole-Brain Functional Networks in Cognitively Normal, Mild Cognitive Impairment, and Alzheimer's Disease. PLoS ONE, 8, e53922. https://doi.org/10.1371/journal.pone.0053922

[11] Biswal, B., Zerrin Yetkin, F., Haughton, V.M. and Hyde, J.S. (1995) Functional 
Connectivity in the Motor Cortex of Resting Human Brain Using Echo-Planar MRI. Magnetic Resonance in Medicine, 34, 537-541. https://doi.org/10.1002/mrm.1910340409

[12] Lee, H., Lee, D.S., Kang, H., Kim, B.-N. and Chung, M.K. (2011) Sparse Brain Network Recovery under Compressed Sensing. IEEE Transactions on Medical Imaging, 30, 1154-1165. https://doi.org/10.1109/TMI.2011.2140380

[13] Friston, K.J., Harrison, L. and Penny, W. (2003) Dynamic Causal Modelling. NeuroImage, 19, 1273-1302. https://doi.org/10.1016/S1053-8119(03)00202-7

[14] Sabilla, S.I., Sarno, R. and Triyana, K. (2019) Optimizing Threshold Using Pearson Correlation for Selecting Features of Electronic Nose Signals. International Journal of Intelligent Systems, 12, 81-90. https://doi.org/10.22266/ijies2019.1231.08

[15] Fornito, A., Zalesky, A. and Bullmore, E. (2016) Fundamentals of Brain Network Analysis. Academic Press, Cambridge.

[16] Yan, C.-G., Wang, X.-D., Zuo, X.-N. and Zang, Y.-F. (2016) DPABI: Data Processing \& Analysis for (Resting-State) Brain Imaging. Neuroinformatics, 14, 339-351. https://doi.org/10.1007/s12021-016-9299-4

[17] Tzourio-Mazoyer, N., et al. (2002) Automated Anatomical Labeling of Activations in SPM Using a Macroscopic Anatomical Parcellation of the MNI MRI Single-Subject Brain. NeuroImage, 15, 273-289. https://doi.org/10.1006/nimg.2001.0978

[18] Peterson, L.E. (2009) K-Nearest Neighbor. Scholarpedia, 4, 1883. https://doi.org/10.4249/scholarpedia.1883

[19] Xu, L., et al. (2016) Prediction of Progressive Mild Cognitive Impairment by Multi-Modal Neuroimaging Biomarkers. Journal of Alzheimer's Disease, 51, 1045-1056. https://doi.org/10.3233/JAD-151010

[20] Wolk, D.A. and Detre, J.A. (2012) Arterial Spin Labeling MRI: An Emerging Biomarker for Alzheimer's Disease and Other Neurodegenerative Conditions. Current Opinion in Neurology, 25, 421. https://doi.org/10.1097/WCO.0b013e328354ff0a 